\title{
Electromyographic, cerebral, and muscle hemodynamic responses during intermittent, isometric contractions of the biceps brachii at three submaximal intensities
}

\author{
Yagesh Bhambhani ${ }^{1 *}$, Jui-Lin Fan ${ }^{2,3}$, Nicolas Place ${ }^{2}$, Javier Rodriguez-Falces ${ }^{4}$ and Bengt Kayser ${ }^{2}$ \\ ${ }^{1}$ Department of Occupational Therapy, Faculty of Rehabilitation Medicine, University of Alberta, Edmonton, AB, Canada \\ ${ }^{2}$ Institute of Sports Sciences and Department of Physiology, Faculty of Biology and Medicine, University of Lausanne, Lausanne, Switzerland \\ ${ }^{3}$ Lemanic Neuroscience Doctoral School, University of Lausanne, Lausanne, Switzerland \\ ${ }^{4}$ Department of Electrical and Electronic Engineering, Public University of Navarra, Pamplona, Spain
}

\section{Edited by:}

Sebastien Racinais, Aspetar, Qatar Orthopaedic and Sports Medicine Hospital, Qatar

\section{Reviewed by:}

Yu-Chieh Tzeng, University of Otago, Wellington, New Zealand Gaël Guilhem, National Institute for Sports (INSEP), France

\section{${ }^{*}$ Correspondence:}

Yagesh Bhambhani, Faculty of Rehabilitation Medicine, University of Alberta, Room 373 Corbett Hall, 8205 - 114 Street, Edmonton, T6G $2 G 4$ AB, Canada e-mail: yagesh.bhambhani@ ualberta.ca
This study examined the electromyographic, cerebral and muscle hemodynamic responses during intermittent isometric contractions of biceps brachii at 20,40, and $60 \%$ of maximal voluntary contraction (MVC). Eleven volunteers completed 2 min of intermittent isometric contractions $(12 / \mathrm{min})$ at an elbow angle of $90^{\circ}$ interspersed with 3 min rest between intensities in systematic order. Surface electromyography (EMG) was recorded from the right biceps brachii and near infrared spectroscopy (NIRS) was used to simultaneously measure left prefrontal and right biceps brachii oxyhemoglobin $\left(\mathrm{HbO}_{2}\right)$, deoxyhemoglobin $(\mathrm{HHb})$, and total hemoglobin $\left(\mathrm{Hb}_{\text {tot }}\right)$. Transcranial Doppler ultrasound was used to measure middle cerebral artery velocity (MCAv) bilaterally. Finger photoplethysmography was used to record beat-to-beat blood pressure and heart rate. EMG increased with force output from 20 to $60 \% \mathrm{MVC}(P<0.05)$. Cerebral $\mathrm{HbO}_{2}$ and $\mathrm{Hb}_{\text {tot }}$ increased while $\mathrm{HHb}$ decreased during contractions with differences observed between $60 \%$ vs. $40 \%$ and $20 \% \operatorname{MVC}(P<0.05)$. Muscle $\mathrm{HbO}_{2}$ decreased while $\mathrm{HHb}$ increased during contractions with differences being observed among intensities $(P<$ 0.05). Muscle $\mathrm{Hb}_{\text {tot }}$ increased from rest at $20 \% \mathrm{MVC}(P<0.05)$, while no further change was observed at 40 and $60 \%$ MVC $(P>0.05)$. MCAv increased from rest to exercise but was not different among intensities $(P>0.05)$. Force output correlated with the root mean square EMG and changes in muscle $\mathrm{HbO}_{2}(P<0.05)$, but not changes in cerebral $\mathrm{HbO}_{2}(P>0.05)$ at all three intensities. Force output declined by $8 \%$ from the 1 st to the 24th contraction only at $60 \% \mathrm{MVC}$ and was accompanied by systematic increases in RMS, cerebral $\mathrm{HbO}_{2}$ and $\mathrm{Hb}_{\text {tot }}$ with a leveling off in muscle $\mathrm{HbO}_{2}$ and $\mathrm{Hb}_{\text {tot. }}$. These changes were independent of alterations in mean arterial pressure. Since cerebral blood flow and oxygenation were elevated at $60 \% \mathrm{MVC}$, we attribute the development of fatigue to reduced muscle oxygen availability rather than impaired central neuronal activation.

Keywords: biceps force, electromyography, cerebral blood flow and oxygenation, muscle blood flow and oxygenation

\section{INTRODUCTION}

Despite the importance of upper limb use in our day-to-day life, particularly during occupational and recreational activities, the underpinning neuromuscular and hemodynamic changes during upper limb muscle contractions at light, moderate, and high intensities have not been well documented. Voluntary muscular force results from cortical neuronal activation leading to motor unit recruitment at the spinal level. Depending on the type (e.g., static or dynamic), intensity, and duration of muscle contraction, the force exerted may be limited by mechanisms at any level, from the cortical neuronal activation to the interaction of contractile proteins in the muscle fibers (Gandevia, 2001; Enoka and Duchateau, 2008). If the contractions are sufficiently intense and/or prolonged, muscle fatigue, defined as a transient loss of force generating capacity, develops (Gandevia, 2001; Enoka and Duchateau, 2008). Classically, muscle fatigue is described as central, when the force generating capacity is impaired through mechanisms proximal to the neuromuscular junction, and peripheral, when distal from the junction (Gandevia, 2001; Enoka and Duchateau, 2008). The extent of central and peripheral fatigue and their interplay depend on the type of muscular activity and the particular circumstances. Surface electromyography (EMG), combined with transcutaneous electrical stimulation, has been extensively used to evaluate the relative contributions of central and peripheral factors limiting muscle force development (Burnley et al., 1985; Gauche et al., 2009; Millet et al., 2011; Neyroud et al., 2013). But despite decades of research, the understanding of the underlying mechanisms remains incomplete.

Near infrared spectroscopy (NIRS) allows simultaneous examination of cerebral and muscular hemodynamic responses [i.e., changes in oxyhemoglobin $\left(\mathrm{HbO}_{2}\right)$ and deoxyhemoglobin 
(HHb)] (Ainslie et al., 2007; Bhambhani, 2012). Several studies have indicated that cerebral tissue desaturation might be a performance-limiting factor during continuous (GonzalezAlonso et al., 2004; Bhambhani et al., 2007; Subudhi et al., 2009; Billaut et al., 2010; Rasmussen et al., 2010) and intermittent (Shibuya et al., 2004a,b) high intensity dynamic exercise involving a large muscle volume (such as running or cycling). It was hypothesized that the reductions in cerebral blood flow, cerebral $\mathrm{HbO}_{2}$, and total hemoglobin $\left(\mathrm{Hb}_{\text {tot }}\right)$, associated to the hyperventilation-induced hypocapnia during heavy exercise may be involved in the disengagement from the effort at the point of exhaustion (Amann et al., 2007; Amann and Kayser, 2009; Subudhi et al., 2009; Rasmussen et al., 2010). In contrast, no cerebral tissue desaturation is observed during exercise involving a smaller muscle volume, such as during unilateral static (Pereira et al., 2007) or dynamic (Matsuura et al., 2011; Gomes et al., 2013) knee extensions. Since both $\mathrm{HbO}_{2}$ and $\mathrm{Hb}_{\text {tot }}$ are compromised at the muscle (Matsuura et al., 2011; Gomes et al., 2013), it is thought that fatigue of the lower limbs during moderate to high resistance is peripherally mediated, in part by limited muscle blood flow and oxygen availability rather than compromised motor drive (Place et al., 2009). In agreement, observations during intermittent isometric knee extensions indicate the presence of a critical intensity threshold, above which fatigue is primarily due to metabolic disturbances in the exercising muscle (Burnley et al., 1985). However, the limiting role of peripheral fatigue for small volume muscle efforts has recently been questioned. Neyroud et al. (2012) found that electrical stimulation at the point of task failure elicited the target force (20\% MVC) during sustained isometric knee extension, which indicates that the lack of force generating capacity associated with peripheral impairment was not responsible for limitation the duration of the task. Instead, they concluded that task failure from a low intensity, sustained isometric contraction was mainly due to central/motivational factors, whereas the MVC force loss was largely explained by the contractile failure of the muscle. Another study by the same group (Neyroud et al., 2013) found that time to task failure during a sustained isometric contraction at $50 \%$ of MVC with the biceps brachii was shorter than that performed with plantar flexors or thumb adductor. While no cerebral or muscle hemodynamic measurements were performed, the researchers speculated that reduced muscle oxygen availability due to restricted blood flow was likely implicated.

To complement these findings we combined surface EMG with cerebral and muscle NIRS to evaluate the neuromuscular and hemodynamic responses during 24 intermittent isometric contractions of the biceps brachii at three submaximal intensities: 20 , 40 , and 60\% MVC. These intensities were selected on the basis of previous studies (Felici et al., 2009; Muthalib et al., 2010), and our pilot data, which suggested that fatigue development could be evidenced at $60 \%$ MVC during this protocol. Research (Maton and Gamet, 1989; Fallentin et al., 1993) has demonstrated that during sustained submaximal contractions up to $80 \%$ MVC, a gradual rise in EMG amplitude normally occurs. This rise in EMG activity is attributed to the recruitment of additional motor units and increased firing rate necessary to counteract the increasing fatigue. Therefore, we hypothesized that during these biceps brachii contractions at 20,40, and 60\% MVC: (1) the root mean square of the EMG (RMS), and the cerebral and muscle hemodynamic responses would change disproportionately to the force output at $60 \% \mathrm{MVC},(2)$ the force output would correlate better with the changes in muscle rather than cerebral hemodynamic responses, and (3) the changes in RMS and cerebral $\mathrm{HbO}_{2}$ would be significantly correlated.

\section{MATERIALS AND METHODS \\ SUBJECTS}

Eleven healthy subjects (nine males and two females) with a mean age of $34.5 \pm 5.2$ years (mean $\pm S D$ ) and body mass index of $24.2 \pm 2.4 \mathrm{~kg} . \mathrm{m}^{-2}$ participated. The local research ethics committee approved the study protocol. The subjects were informed regarding the procedures, and signed consent was given prior to participation. All subjects were recreationally active but none of them were involved in systematic resistance training programs during the course of the study.

\section{TEST PROTOCOL}

Each subject completed a single testing session using a custom built ergometer equipped with an adjustable handle and strain gauge as previously described (Neyroud et al., 2013). After a short warm-up consisting of 8-10 submaximal isometric contractions at approximately $50 \%$ of estimated MVC, the subject performed three MVCs by exerting maximal force with the right forearm at an elbow angle of $90^{\circ}$. Contractions were interspersed with $30 \mathrm{~s}$ rest to facilitate sufficient recovery. The highest of the three force values was retained as MVC force. The subjects then did 24 intermittent isometric contractions of the biceps brachii at 20, 40 , and $60 \%$ MVC in systematic order, at 12 contractions per minute ( $2.5 \mathrm{~s}$ contraction and $2.5 \mathrm{~s}$ relaxation) in rhythm with a metronome. The average force output of the 24 contractions was expressed as a percentage of MVC. Pilot testing indicated that a 3-min rest interval between the three intensities was sufficient to minimize the effects of fatigue and allow the cerebral and muscle hemodynamic responses to recover to baseline values. Magnetic resonance spectroscopy findings indicate near maximal resynthesis of intramuscular phosphocreatine and ATP occurs after $3 \mathrm{~min}$ of rest following high intensity exercise (Prompers et al., 2014). The subjects were instructed to exhale during the contractions and inhale during relaxation so as to avoid any Valsalva maneuvers at the higher intensities, which could influence the cerebral hemodynamic measurements (Pott et al., 2003).

\section{PHYSIOLOGICAL MEASUREMENTS}

All signals were acquired using an analog-to-digital converter (PowerLab 16/35, ADInstruments, Australia) with commercially available software (LabChart, version 7.2, ADInstruments, Australia), and stored on disk for subsequent analysis.

\section{Biceps brachii performance}

Force output of the elbow flexors was recorded at $2 \mathrm{kHz}$ using a custom designed ergometer with the right forearm in the verti$\mathrm{cal}$ and supinated position and the elbow flexed at $90^{\circ}$. The seat height was adjusted so that the shoulder was in line with the base 
of the ergometer, which was mounted on a tabletop. The subject exerted force against the ergometer handle, which was fitted with a strain gauge (SAS $200 \mathrm{~kg}$, sensitivity $1.998 \mathrm{mV} / \mathrm{V}$, SWJ, China). The ergometer was interfaced with the AD-system (PowerLab 16/35, ADInstruments, Australia) so that the desired force output at the three intensities could be displayed on a computer monitor for visual feedback to the subject.

\section{Electromyography recordings}

The EMG activity of the biceps brachii muscle was recorded with pairs of silver chloride $(\mathrm{Ag} / \mathrm{AgCl})$ circular (recording diameter of $1 \mathrm{~cm}$ ) surface electrodes (Kendall Meditrace 100, Tyco, Canada) positioned lengthwise over the middle of the muscle belly with an inter-electrode (center-to-center) distance of $2 \mathrm{~cm}$. The reference electrode was placed over the ipsilateral wrist. The electrode site was shaved lightly abrading the skin and cleaning with alcohol to minimize electrical resistance (verified $<10 \mathrm{k} \Omega$ ). The myoelectrical signals were amplified with a gain of 1000 and filtered using a bandwidth with frequency between 10 and $500 \mathrm{~Hz}$ (LabChart version 7.2, ADInstruments, Australia). The filtered EMG signals were sampled at $2 \mathrm{kHz}$ by the analog-to-digital converter. A common mode rejection ratio of $90 \mathrm{~dB}$ was used to supress extraneous noise and enhance the signal to noise ratio. For each MVC, the RMS was quantified during the period (approximately $0.5 \mathrm{~s}$ ) where the peak force was developed. For the submaximal contractions at each intensity, the RMS was quantified during the force plateau (approximately $1 \mathrm{~s}$ ) observed during individual contractions.

\section{Cerebrovascular and cardiovascular measurements}

Bilateral middle cerebral artery velocities (MCAv, as an index of cerebral blood flow, $\mathrm{CBF}$ ) were measured using a $2-\mathrm{MHz}$ pulsed Doppler ultrasound system (ST3 Spencer technology, USA). The probes were positioned over the temporal windows and firmly held in place with an adjustable headband. The signals were recorded at depths ranging from 43 to $54 \mathrm{~mm}$. The bilateral MCAv were averaged to represent global CBF during rest and exercise. Beat-to-beat systolic (SBP), diastolic (DBP), and mean arterial blood pressure (MAP) was monitored with finger photoplethysmography (Finometer Midi, Finapress Medical Systems, Netherlands) using standardized procedures (Ainslie et al., 2007).

\section{Cerebral and muscle hemodynamic using NIRS}

Cerebral and muscle $\mathrm{HbO}_{2}$ and $\mathrm{HHb}$ were recorded simultaneously during the baseline, exercise and recovery phases using continuous dual-wavelength NIRS (Oxymon, Artinis Medical Systems, Netherlands). The cerebral probe was modified to fit on the adjustable headband used to record the Doppler blood flow measurements (see above). The probe was placed on the left prefrontal lobe approximately $3 \mathrm{~cm}$ from the midline of the forehead, just above the supraorbital ridge (Bhambhani et al., 2006; Rasmussen et al., 2007). The muscle probe was placed adjacent to the EMG electrodes over the belly of the right biceps brachii to ensure metabolic homogeneity (Pappas et al., 2002; Felici et al., 2009). It was secured with a dark tensor bandage to minimize artifact from stray light. Prior to each test, the cerebral and muscle NIRS probes were calibrated according to the manufacturer's instructions. All signals were recorded at $50 \mathrm{~Hz}$ and stored for subsequent analysis. The sum of $\mathrm{HbO}_{2}$ and $\mathrm{HHb}$ was used to calculate $\mathrm{Hb}_{\text {tot }}$, which was considered an index of localized blood flow in cerebral and muscle tissue (Boushel et al., 2000). The difference between $\mathrm{HbO}_{2}$ and $\mathrm{HHb}\left(\mathrm{Hb}_{\text {diff }}\right)$ was used as an index of tissue oxygen extraction. Delta values of these NIRS variables were calculated as follows: peak value attained at each exercise intensity minus the baseline value prior to the onset of exercise. The baseline was the average 20-s value between 2:30 to 2:50 of the 3 min resting period prior to the onset of each exercise intensity. The final $10 \mathrm{~s}$ were not used in calculating the baseline value because anticipation of exercise can alter the hemodynamic responses (Colier et al., 1995).

\section{STATISTICAL ANALYSIS}

Normality of the data was initially verified using the Shapiro-Wilk test. Thereafter, One-Way repeated measures analysis of variance (ANOVA) was used to examine the differences in the group mean values of the physiological responses at 20, 40, and 60\% MVC. Two-Way repeated measures analysis of covariance (ANCOVA) was used to examine the changes in the physiological responses during the transition from the 1st to the 24th contraction at 20, 40 , and $60 \%$ MVC, with MAP as a covariate. Significant 'F' ratios were analyzed on a post-hoc basis using the Scheffe procedure for multiple comparisons. The Bonferroni correction procedure was applied to minimize the possibility of Type 1 error. Pearson correlations were used to examine relationships between force output and the RMS, cerebral and muscle hemodynamic variables at 20,40, and 60\% MVC. Values were considered significant at $P<0.05$. All statistical analyses were performed using SPSS software (version 14.1).

\section{RESULTS}

\section{FORCE OUTPUT AND ELECTROMYOGRAPHIC RESPONSES AT THE THREE INTENSITIES}

The mean values of the force output and RMS for the 24 intermittent isometric contractions of the biceps brachii at 20, 40, and $60 \%$ MVC are summarized in Table 1. The increase in force output across the three intensities was accompanied by significant increases in the RMS, both in absolute values and relative to the MVC. This was observed in all subjects across all exercise intensities, but with considerable inter-subject variability.

\section{CEREBROVASCULAR AND CARDIOVASCULAR RESPONSES AT THE THREE INTENSITIES}

The mean values of the cerebrovascular and cardiovascular responses at the three intensities are summarized in Table 1. The right and left MCAv increased from the baseline value at all three intensities, with no differences observed among the intensities. Likewise, no differences were observed between the right and left MCAv during these contractions at all exercise intensities. MAP increased significantly above baseline, but there were no differences among the intensities. The increase in MAP was due to increases in both SBP and DBP during the biceps brachii contractions. Heart rate increased above the resting value and demonstrated a systematic increase across the three intensities. 
Table 1 | Electromyographic, cardiovascular and cerebrovascular responses at rest and during 24 intermittent isometric contractions of the biceps brachii at three intensities (Values are mean $\pm S D, N=11$ ).

\begin{tabular}{|c|c|c|c|c|}
\hline Variable & Rest & $20 \% \mathrm{MVC}^{\dagger}$ & $40 \% \mathrm{MVC}^{\dagger}$ & $60 \% \mathrm{MVC}^{\dagger}$ \\
\hline $\mathrm{RMS},(\mathrm{mV})$ & - & $0.18 \pm 0.11$ & $0.43 \pm 0.28^{*}$ & $0.77 \pm 0.41^{\ddagger}$ \\
\hline Mean MCAv, $\left(\mathrm{cm} \cdot \mathrm{s}^{-1}\right)^{a}$ & $36.5 \pm 7.9$ & $49.8 \pm 11.2$ & $46.8 \pm 10.3$ & $49.3 \pm 11.2$ \\
\hline $\mathrm{MAP}(\mathrm{mmHg})$ & $76.6 \pm 9.9$ & $86.6 \pm 14.9$ & $83.6 \pm 19.3$ & $91.8 \pm 23.4$ \\
\hline $\mathrm{HR}(\mathrm{bpm})$ & $62.7 \pm 6.5$ & $75.2 \pm 29.4$ & $91.5 \pm 23.1^{*}$ & $100.1 \pm 23.0^{\ddagger}$ \\
\hline
\end{tabular}

${ }^{a}$ Mean MCAv is the average of the right and left values as there were no significant differences between the two sides.

${ }^{\dagger}$ All exercise values shown are significantly different from the corresponding resting values "* indicates significantly different from mean value at $20 \%$ MVC.

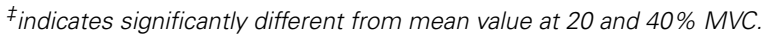

\section{CEREBRAL AND MUSCLE HEMODYNAMIC RESPONSES AT THE THREE INTENSITIES}

The mean delta values of the cerebral and muscle hemodynamic responses are illustrated in Figures 1, 2, respectively. Cerebral $\mathrm{HbO}_{2}, \mathrm{Hb}_{\text {diff, }}$, and $\mathrm{Hb}_{\text {tot }}$ increased while $\mathrm{HHb}$ decreased with increasing intensity. There was no difference between 40 and $60 \%$ MVC for cerebral $\mathrm{HHb}$. Muscle $\mathrm{HbO}_{2}, \mathrm{Hb}_{\text {tot }}$, and $\mathrm{Hb}_{\text {diff }}$ declined while $\mathrm{HHb}$ increased with increasing intensity. $\mathrm{Hb}_{\text {tot }}$ was higher at $20 \%$ MVC compared to 40 and $60 \%$ MVC but was not different between 40 and 60\% MVC.

\section{RELATIONSHIPS BETWEEN FORCE OUTPUT, ELECTROMYOGRAPHIC, AND HEMODYNAMIC RESPONSES}

Pearson correlations summarizing the relationships between force output, RMS, cerebral and muscle hemodynamic responses at the three intensities are presented in Table 2. The correlations between force output and RMS were significant at each intensity with a trend toward a stronger relationship at 20 and $40 \%$ MVC compared to $60 \% \mathrm{MVC}$. Force output was not significantly correlated with cerebral $\mathrm{HbO}_{2}$ and $\mathrm{HHb}$ at any intensity. However, it was significantly correlated with muscle $\mathrm{HbO}_{2}$ and was approaching significance with $\mathrm{HHb}$ at all three intensities. The correlations between RMS and cerebral as well as muscle $\mathrm{HbO}_{2}$ and $\mathrm{HHb}$ changes during the intermittent biceps brachii contractions at the three intensities are summarized in Table 3. RMS was not significantly correlated with the cerebral or muscle $\mathrm{HbO}_{2}$ and $\mathrm{HHb}$ at any of the intensities.

\section{TRANSITIONAL CHANGES IN FORCE OUTPUT, ELECTROMYOGRAPHIC, AND HEMODYNAMIC RESPONSES AT THE THREE INTENSITIES}

The changes in mean force output and RMS during the transition from the 1st to the 24th contraction at each intensity are illustrated in Figures 3A,B, respectively. At 20 and 40\% MVC, there were no changes in the force output and RMS from the 1 st to the 24 th contraction. However, at $60 \%$ MVC force output declined by $8 \%$ from the 1st to the 12th and 24th contractions and was unchanged between the 12th and 24th contractions. This decline in force output was accompanied by an increase in RMS. Increases in MCAv and MAP were observed between the 1 st to the 12th contraction, but not from the 12th to the 24th contraction at each intensity (Figures 3 C,D, respectively). The changes in cerebral and muscle $\mathrm{HbO}_{2}$ and $\mathrm{Hb}_{\text {tot }}$ are illustrated in
Figures 4A-D, respectively. Cerebral $\mathrm{HbO}_{2}$ and $\mathrm{Hb}_{\text {tot }}$ increased progressively from the 1 st to the 24th contraction with no sign of leveling off. In contrast, muscle $\mathrm{HbO}_{2}$ and $\mathrm{Hb}_{\text {tot }}$ demonstrated a decline from the 1st to the 12th contraction and a plateau during the remaining 12 contractions. A representative trend is available in Figures 5A-C.

\section{DISCUSSION}

This study used a multi-modal, non-invasive approach to simultaneously evaluate the electromyographic, cerebrovascular and hemodynamic responses during intermittent, isometric elbow flexor contractions at 20,40, and 60\% MVC. Before discussing the physiological implications of our findings, we first address some of the methodological considerations of these non-invasive techniques.

\section{METHODOLOGICAL CONSIDERATIONS}

Electromyography in conjunction with NIRS is used to noninvasively evaluate cerebral and muscle activation during exercise involving large muscle volume. Alterations in RMS are thought to reflect changes in central activation influencing force output during various types of muscle contractions. However, EMG signals do not allow us to differentiate between the supraspinal (cortical) and spinal contributions to muscle activation (Burnley et al., 1985; Millet et al., 2011). Meanwhile, the validity of cerebral NIRS for assessing cortical oxygenation status has been compared with MRI measurements (Huppert et al., 2006; Schroeter et al., 2006). An increase in cerebral $\mathrm{HbO}_{2}$ with a concomitant decline in $\mathrm{HHb}$ reflects enhanced neuronal activation via neurovascular coupling (Rasmussen et al., 2010). Such hemodynamic responses are accompanied by enhanced oxygen, glucose and lactate utilization, which reflects increased metabolic needs of the activated neurons (Dalsgaard and Secher, 2007). Given the close relationship between measurements from the left prefrontal lobe and the motor cortex, prefrontal lobe changes are used as a surrogate for alterations in the motor cortex (Ainslie et al., 2007; Subudhi et al., 2009). Nevertheless, our cortical tissue measurements should be interpreted with caution as prefrontal NIRS signals can be influenced by changes in scalp blood flow (Payne et al., 2011). While the decline in $\mathrm{HbO}_{2}$ accompanied by a plateau or increase in $\mathrm{HHb}$ indicates enhanced oxidative metabolism of contracting fibers (Mancini et al., 1994; Bhambhani, 2012), the muscle NIRS signals 


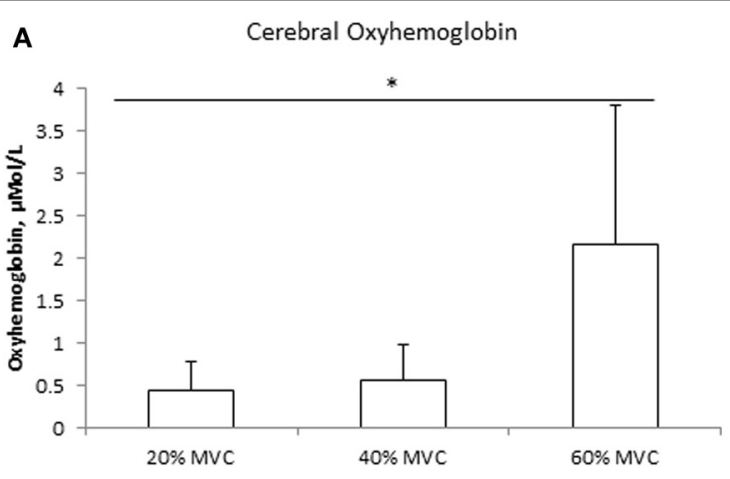

C

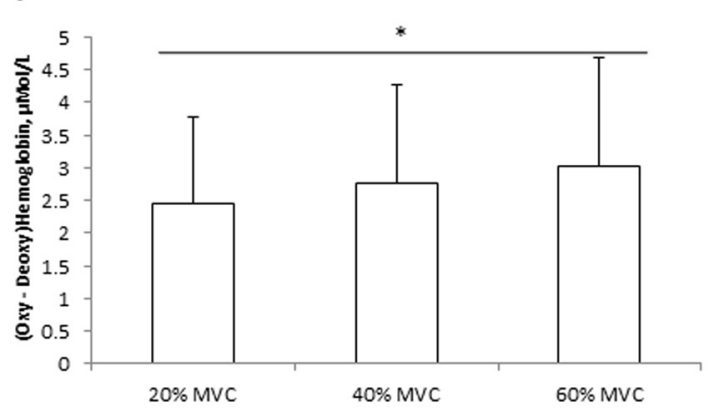

B

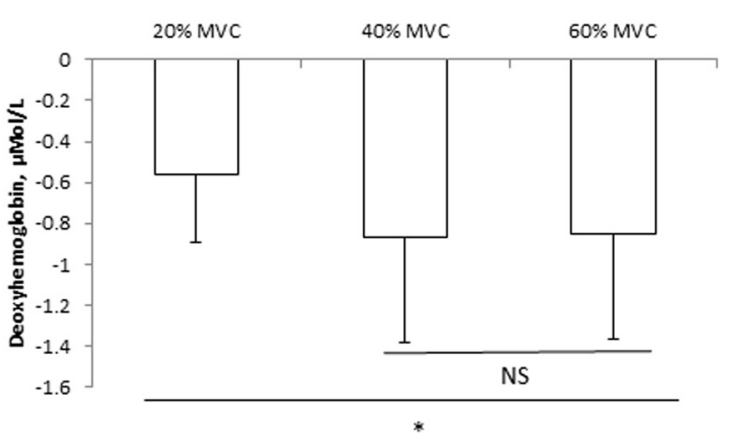

D

Cerebral Total Hemoglobin

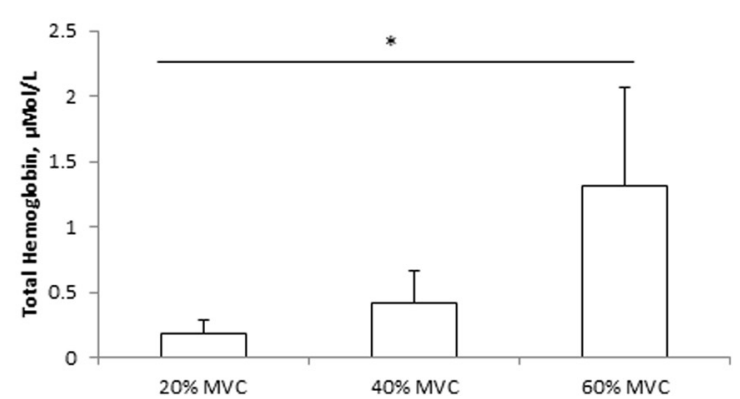

FIGURE 1 | Cerebral hemodynamic responses at three different intensities of intermittent isometric contractions of the biceps brachii. *Indicates $P<0.05$.

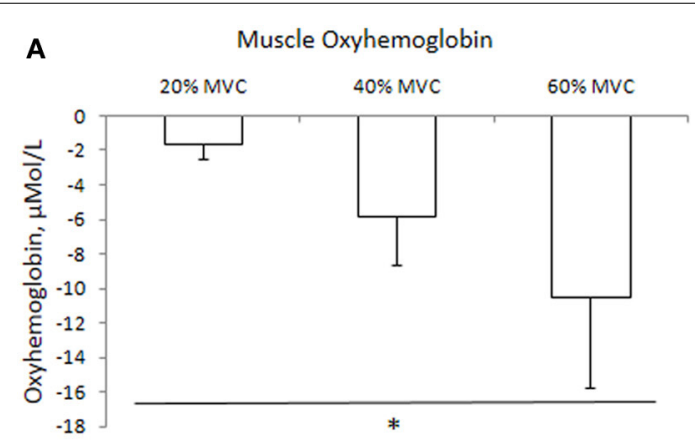

C

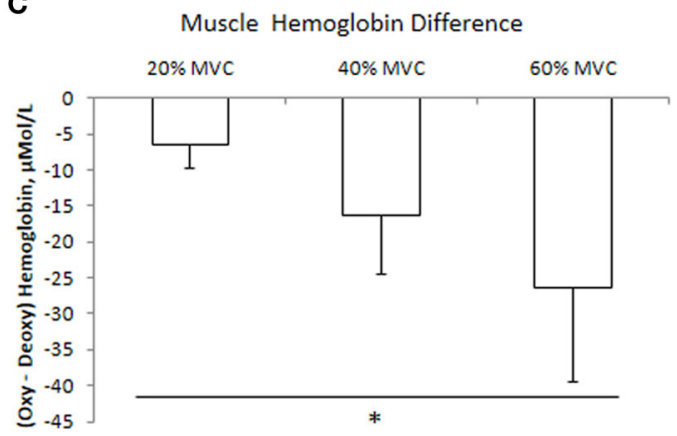

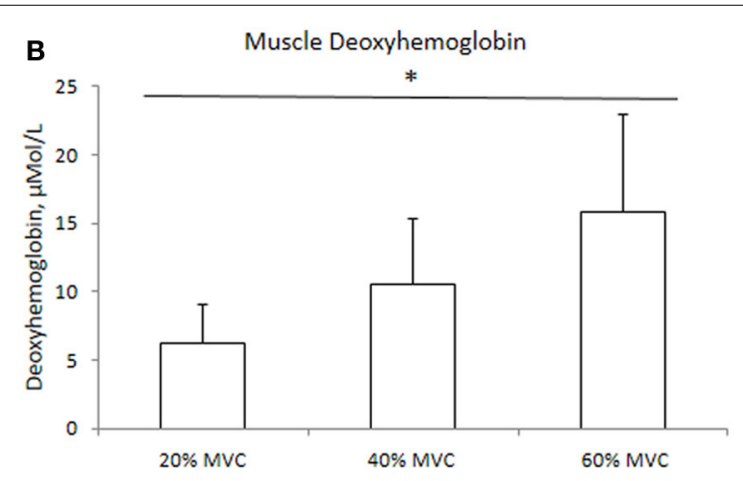

D

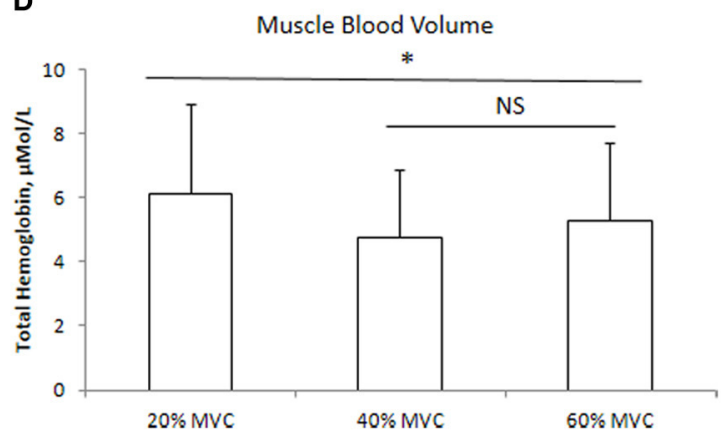

FIGURE 2 | Muscle hemodynamic responses at three different intensities of intermittent isometric contractions of the biceps brachii. 
Table 2 | Pearson correlations and common variance (in parentheses) between force output and selected electromyographic, cerebral, and muscle hemodynamic variables during intermittent contractions of the biceps brachii at three intensities ( $N=11$, critical ' $r$ ' value $=0.58$ ).

\begin{tabular}{|c|c|c|c|}
\hline \multirow[t]{2}{*}{ Variable } & \multicolumn{3}{|c|}{$\begin{array}{c}\text { Pearson } r\left(\% r^{2}\right) \text { between force output and } \\
\text { variables at three intensities }\end{array}$} \\
\hline & $20 \%$ MVC & $40 \%$ MVC & $60 \%$ MVC \\
\hline RMS & $0.73 *(53.6 \%)$ & $0.71 *(50.2 \%)$ & $0.59 *$ \\
\hline Delta cerebral $\mathrm{HbO}_{2}$ & $0.46(21.4 \%)$ & $0.19(3.1 \%)$ & $0.24(5.6 \%)$ \\
\hline Delta cerebral HHb & $0.35(12.2 \%)$ & $0.33(10.9 \%)$ & $0.04(1.8 \%)$ \\
\hline Delta muscle $\mathrm{HbO}_{2}$ & $0.64 *(41.0 \%)$ & $0.62 *(38.4 \%)$ & $0.63 *(39.8 \%)$ \\
\hline Delta muscle $\mathrm{HHb}$ & $0.54(29.5 \%)$ & $0.57(32.5 \%)$ & $0.51(26.0 \%)$ \\
\hline
\end{tabular}

${ }^{*}$ Correlation significant at $P<0.05$. Note: force output was significantly correlated with RMS at 20 and 40\% MVC but not at 60\% MVC. Force output was significantly correlated with muscle $\mathrm{HbO}_{2}$ but not cerebral $\mathrm{HbO}_{2}$, cerebral $\mathrm{HHb}$ and muscle $\mathrm{HHb}$.

Table 3 | Pearson correlations between electromyographic and cerebral/muscle hemodynamic responses during intermittent contractions of the biceps brachii at three intensities $(N=11$, critical ' $r$ ' value $=0.58$ ).

\begin{tabular}{lcccc}
\hline Intensity & $\begin{array}{c}\text { Cerebral } \\
\mathbf{H b O}_{\mathbf{2}}\end{array}$ & $\begin{array}{c}\text { Cerebral } \\
\mathbf{H H b}\end{array}$ & $\begin{array}{c}\text { Muscle } \\
\mathbf{H b O}_{\mathbf{2}}\end{array}$ & $\begin{array}{c}\text { Muscle } \\
\mathbf{H H b}\end{array}$ \\
\hline RMS at 20\% MVC & 0.44 & 0.03 & -0.16 & 0.25 \\
RMS at 40\% MVC & 0.51 & -0.05 & -0.19 & 0.09 \\
RMS at 60\% MVC & 0.49 & -0.11 & -0.25 & 0.16 \\
\hline
\end{tabular}

None of the correlations were significant at $P<0.05$.

are directly influenced by adipose tissue thickness (van Beekvelt et al., 2001). However, representative measurements are obtained in subjects with a BMI less than 32 (McCully and Hamaoka, 2000); a criterion met by all the subjects in this study. Transcranial Doppler ultrasound measures blood flow velocity per se, rather than flow. While the MCAv changes during dynamic incremental exercise have been shown to reflect changes in internal carotid blood flow (Sato et al., 2011), we cannot exclude the possibility that the MCA diameter increased during isometric contraction, which would lead to an underestimation of CBF changes. Finally, this study only assessed the RMS and hemodynamic responses of the biceps brachii. It should be recognized that the brachialis and brachioradialis muscles are also activated during sustained elbow flexion, with differential load sharing patterns during submaximal isometric contractions (Bouillard et al., 2012). If such a similar load-sharing pattern occurred during the intermittent isometric contractions in this study, the muscle hemodynamic responses may have differed among these three muscles and altered the interpretation of these results.

\section{ELECTROMYOGRAPHIC RESPONSES AT THE THREE INTENSITIES}

The increased force output of the biceps brachii during the intermittent isometric contractions at the three intensities was accompanied by an increase in biceps brachii RMS expressed in absolute values or relative to the MVC (Table 1). Although there was a tendency for RMS to increase disproportionately at $60 \%$ MVC compared to 20 and $40 \%$ MVC, these differences were not statistically significant. This was most likely due to the fact that we did not exercise the subjects to voluntary fatigue, but terminated the test after a finite number of muscle contractions (Felici et al., 2009) in our protocol. As well, the contractions were intermittent in nature as supposed to being sustained, which would have enabled some recovery in the neuromuscular responses between the successive contractions. The increase in RMS implies enhanced motor unit firing frequency and/or greater motor unit recruitment to generate the desired force output at the higher exercise intensities (Felici et al., 2009; Neyroud et al., 2013). In accordance with the Henneman size principle (Henneman et al., 1965), it is likely that there was a systematic progression in the recruitment starting with the Type 1, low activation threshold (slow twitch) motor units, followed by inclusion of the fastfatigable Type II motor units, in order to develop the desired force at the higher intensities.

\section{CEREBROVASCULAR RESPONSES AT THE THREE INTENSITIES}

Mean MCAv increased from the resting baseline value at all three intensities during the unilateral intermittent contractions of the biceps brachii (Table 1). This observation is consistent with previous reports of increased MCAv during rhythmic and sustained hand grip contractions at submaximal and maximal intensities (Giller et al., 2000; Zebrowska et al., 2013)—presumably due to increased central command (Vianna et al., 2009). However, since there is considerable heterogeneity in MCAv responses during maximal intermittent isometric exercise between individuals, with some subjects demonstrating an increase and others showing a decrease, these findings should be viewed with caution (Giller et al., 2000).

One of the novel findings of the present study was that the rise in MCAv during isometric contraction was the same between different contraction intensities, with no difference between the hemispheres, despite an increase in heart rate (Table 1). We attribute this lack of difference in MCAv rise between contraction intensities to the: (1) intermittent nature of the contractions (i.e., $2.5 \mathrm{~s}$ contraction followed by $2.5 \mathrm{~s}$ relaxation); and (2) avoidance of the Valsalva maneuver by exhaling during the contractions. Both these factors would have attenuated the expected blood pressure rise and associated increase in MCAv during the contraction period (Haykowsky et al., 2003; Zebrowska et al., 2013). In support, we found no difference in MAP among the three intensities (Table 1). We found the increase in prefrontal $\mathrm{Hb}_{\text {tot }}$ was greater at higher exercise intensity (Figure 2D). Since this occurred in the absence of a greater increase in MCAv (Table 1), we speculate that this increase in $\mathrm{Hb}_{\text {tot }}$ was likely due to a redistribution of the CBF increase during isometric elbow flexion. Taken together, these findings indicate that, when controlled for MAP, global CBF increases by the same extent during low, moderate and heavy intermittent isometric contractions.

\section{CEREBRAL AND MUSCLE HEMODYNAMIC RESPONSES AT THE THREE INTENSITIES}

Consistent with our hypothesis, mean cerebral $\mathrm{HbO}_{2}$ increased while $\mathrm{HHb}$ decreased progressively with increasing exercise 

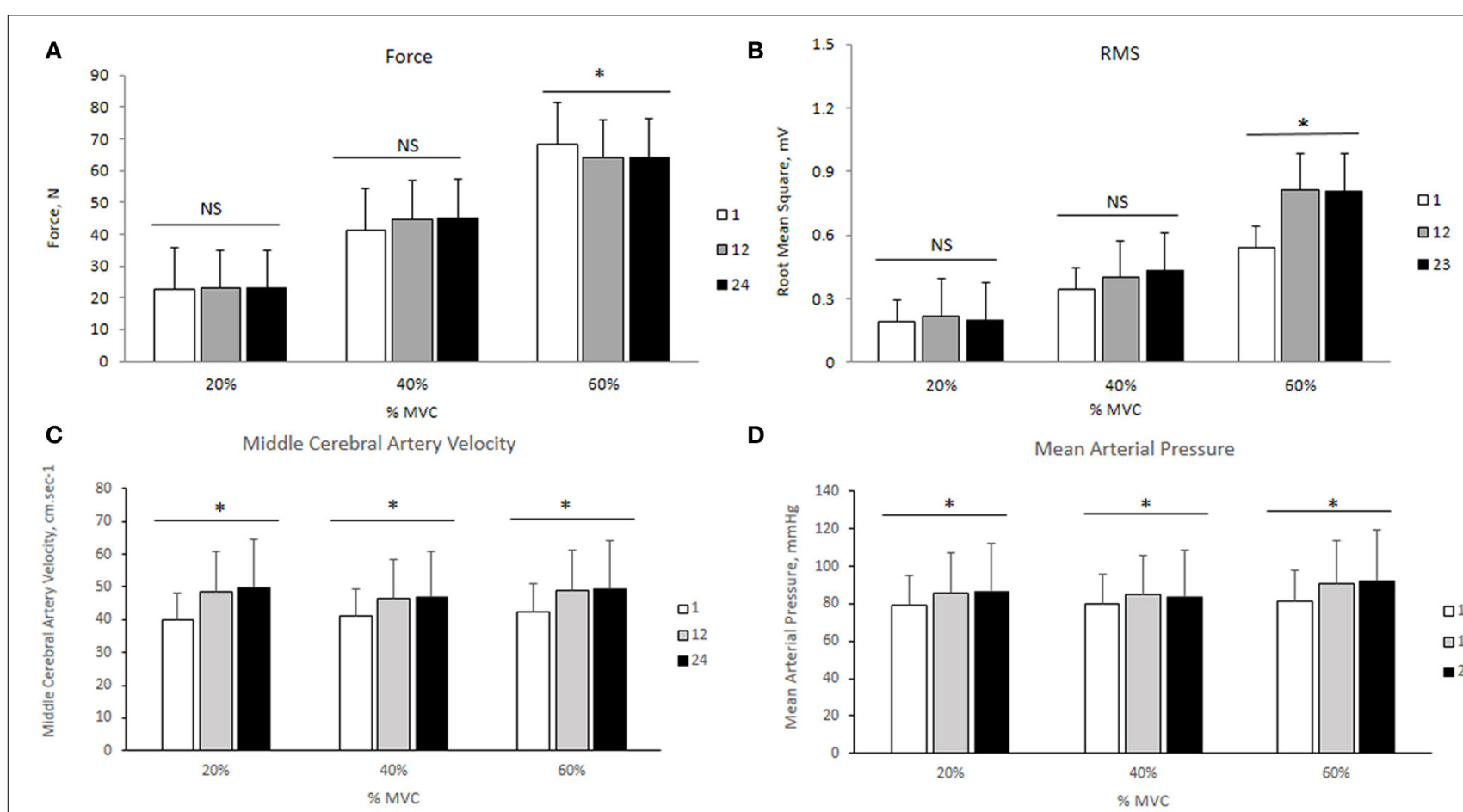

D

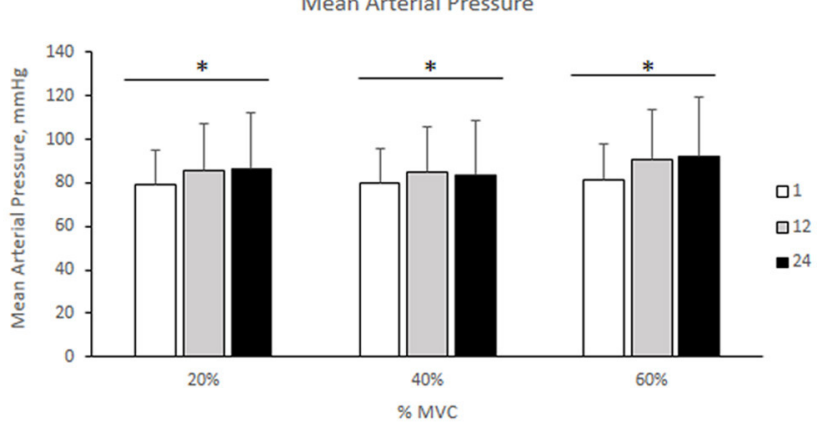

FIGURE 3 | Force output, root mean square, middle cerebral artery velocity, and mean arterial pressure during 24 intermittent isometric contractions of the biceps brachii at three different intensities. ${ }^{*}$ Indicates $P<0.05$.

intensity (Figures 1A,B), independent of changes in MAP. These trends are consistent with previous measurements during intermittent isometric elbow flexion performed at 30 and $100 \%$ MVC (Muthalib et al., 2012) and during repeated maximal hand grip contractions (Bhambhani et al., 2006). The increases in cerebral $\mathrm{HbO}_{2}$ and $\mathrm{Hb}_{\text {diff, coupled with the concomitant decrease }}$ in $\mathrm{HHb}$ suggest enhanced neuronal activation (Dalsgaard and Secher, 2007; Ogoh and Ainslie, 2009). This supports our EMG observations of increased RMS (as an index of neural drive) with increasing intensity from 20 to $60 \%$ MVC. Therefore, our findings indicate that the increased motor-neuronal activation and associated increased motor recruitment with progressively higher exercise intensities can be observed with our cerebral NIRS measurements.

Using positron emission tomography, Korotkov et al. (2005) reported increased regional CBF during sustained isometric elbow flexion at $30-50 \%$ MVC. Specifically, they found blood flow to the primary and secondary somatosensory areas, the somatosensory association area, and the temporal area contralateral to the muscle, to increase with increasing intensity and duration of the fatiguing contractions. Furthermore, they found these regional CBF increases were associated with enhanced cortical activation, which spread to several cortical areas and reflected the changes in both excitatory and inhibitory cortical circuits. In the present study, we found increases in cerebral $\mathrm{HbO}_{2}$ and $\mathrm{Hb}_{\text {tot }}$ were disproportionately greater, relative to force output, at $60 \%$ MVC (Figure 1D). We speculate this disproportionate increased prefrontal activation and blood flow might be due to reduced efficiency during high intensity contractions. In support, visual observation indicated that the subjects began to recruit accessory muscle, such as the trunk and shoulder muscles, to maintain the target force for this intensity. This would lead to additional neuronal activation, which may account for the exaggerated increase in cerebral $\mathrm{HbO}_{2}$ observed at $60 \%$ MVC.

In agreement with previous research (Felici et al., 2009; Muthalib et al., 2010a,b), muscle $\mathrm{HbO}_{2}$ decreased with increasing intensity while muscle $\mathrm{HHb}$ increased (Figures 3A,B). Praagman et al. (2003) reported a significant relationship between force output and muscle oxygenation during sustained isometric elbow flexion up to $70 \%$ MVC. Similarly, we found a strong relationship between these two variables between 20 and 60\% MVC (Table 2). We interpret this as increased oxygen extraction in order to meet the increased aerobic ATP production during effort and associated increase in motor unit recruitment. It should be noted that these trends in $\mathrm{HbO}_{2}$ and $\mathrm{HHb}$ were evident during the entire sequence of contractions (from 1 to 24), even though they were intermittent in nature. Perhaps surprisingly, we observed no reoxygenation during the $2.5 \mathrm{~s}$ relaxation periods between the contractions. We speculate that this might reflect the restoration of oxymyoglobin and phosphocreatine in the muscle (Kime et al., 2003). During exercise, the increase in $\mathrm{Hb}_{\text {tot }}$ was higher at $20 \%$ MVC compared to 40 and $60 \%$ MVC (Figure 2D). We attribute this to a lower intramuscular pressure during the contraction and relaxation phase at $20 \% \mathrm{MVC}$, which allows for better reperfusion of the tissue, thereby increasing the muscle blood measured by NIRS. Since the $\mathrm{Hb}_{\text {tot }}$ increase was not different between 40 and $60 \%$ MVC, we speculate that a threshold intramuscular pressure for blood was attained above $20 \%$ MVC. This is consistent 


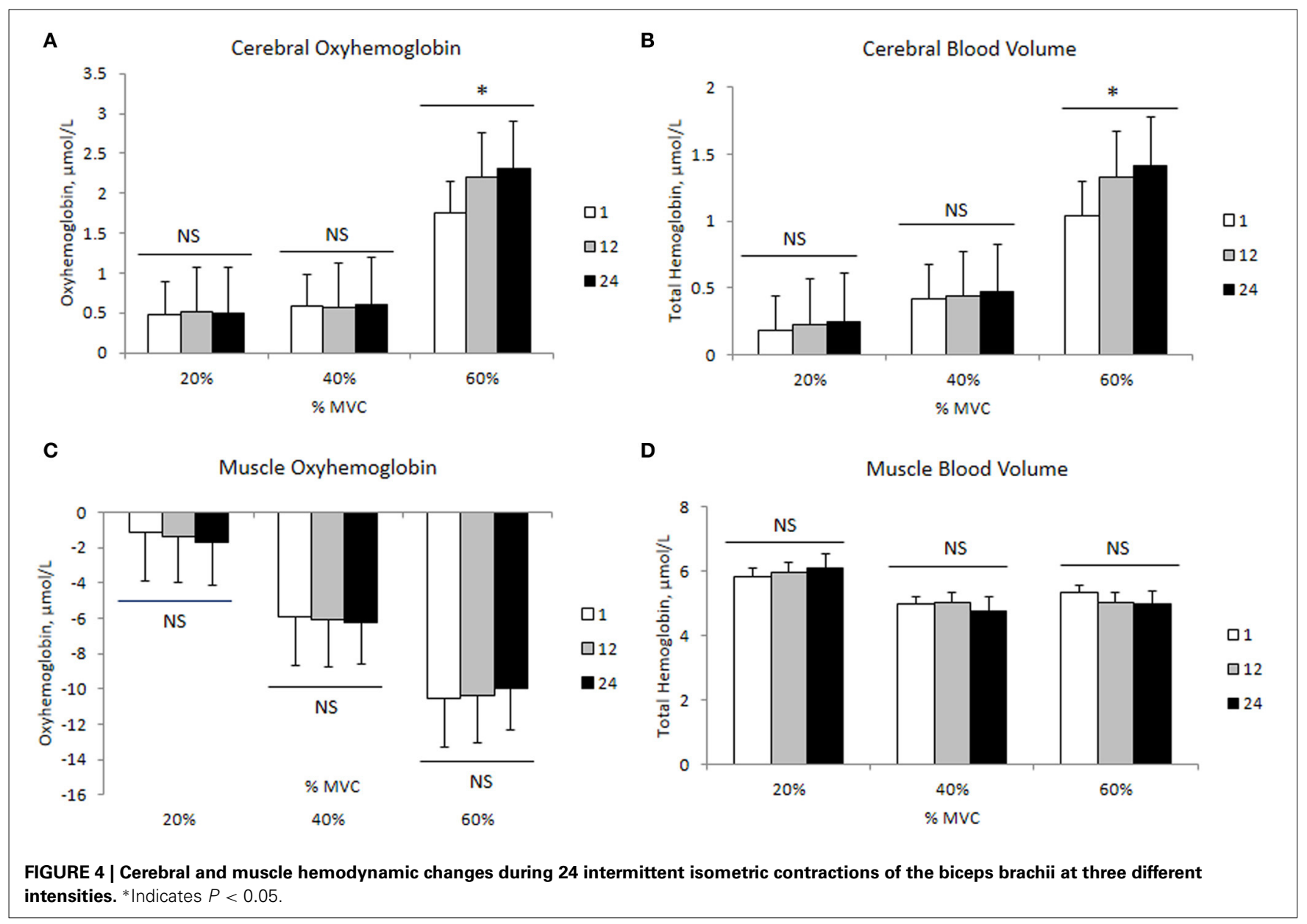

with a previous study (Zwarts and Arendt-Nielsen, 1988) which found muscle blood flow to be reduced during sustained elbow flexion at 50-60\% MVC, but not at 10\% MVC.

\section{RELATIONSHIP BETWEEN NEUROMUSCULAR AND HEMODYNAMIC RESPONSES}

In contrast to our hypothesis, we found no correlation between the RMS with either the cerebral or muscle hemodynamic responses $\left(\mathrm{HbO}_{2}\right.$ and $\left.\mathrm{HHb}\right)$ at any of the exercise intensities (Table 3). Our findings contrast those by Felici et al. (2009) which reported a significant relationship between RMS and muscle tissue oxygen saturation (ratio between $\mathrm{HbO}_{2}$ and $\mathrm{Hb}_{\text {tot }}$ ) during sustained elbow flexion at $20-80 \%$ MVC. From this, they concluded that these two techniques provided complementary information pertaining to motor unit recruitment and muscle oxidative metabolism. The lack of a significant relationship between EMG and NIRS results in the present study could be due to the large inter-individual variability of these responses and the limited number of participants.

TRANSITIONAL CHANGES IN FORCE OUTPUT, ELECTROMYOGRAPHIC, AND HEMODYNAMIC RESPONSES AT THE THREE INTENSITIES

We observed a significant decline in force output between the 1 st to the 24th intermittent isometric contraction at $60 \% \mathrm{MVC}$ while no such decline was seen at 20 and $40 \%$ MVC. Since RMS, cerebral $\mathrm{HbO}_{2}$ and $\mathrm{Hb}_{\text {tot }}$ showed a consistent increase during this transition, while a plateau was observed in both MCAv and MAP, it seems unlikely that this decline in force output was due to a reduction in neuronal activation. Instead, we believe the increase in RMS, in the face of declining force output, may be attributed to: (1) greater recruitment of fast but more fatigable motor units (Type II) (Gandevia, 2001; Enoka and Duchateau, 2008), (2) increased synchronization of motor units (Gandevia, 2001; Enoka and Duchateau, 2008), and (3) slowing of muscle fiber action potential conduction velocity in order to meet the force demands (Lindstrom et al., 1970). Neyroud et al. (2012) proposed that during a sustained isometric contraction of the biceps brachii at 50\% MVC, the development of peripheral muscle fatigue was due to reduced muscle blood flow and oxygen availability. The data from the present study appears to give support to this hypothesis. Recently it was suggested that during intermittent isometric knee extension to exhaustion a critical threshold exists, above which the decline in force output was primarily induced by peripheral metabolic alterations rather than central neuronal activation (Burnley et al., 1985). Our results support the existence of such a threshold during intermittent elbow flexion, a hypothesis which should be tested further. Our findings are of potential use in developing scientifically sound exercise 

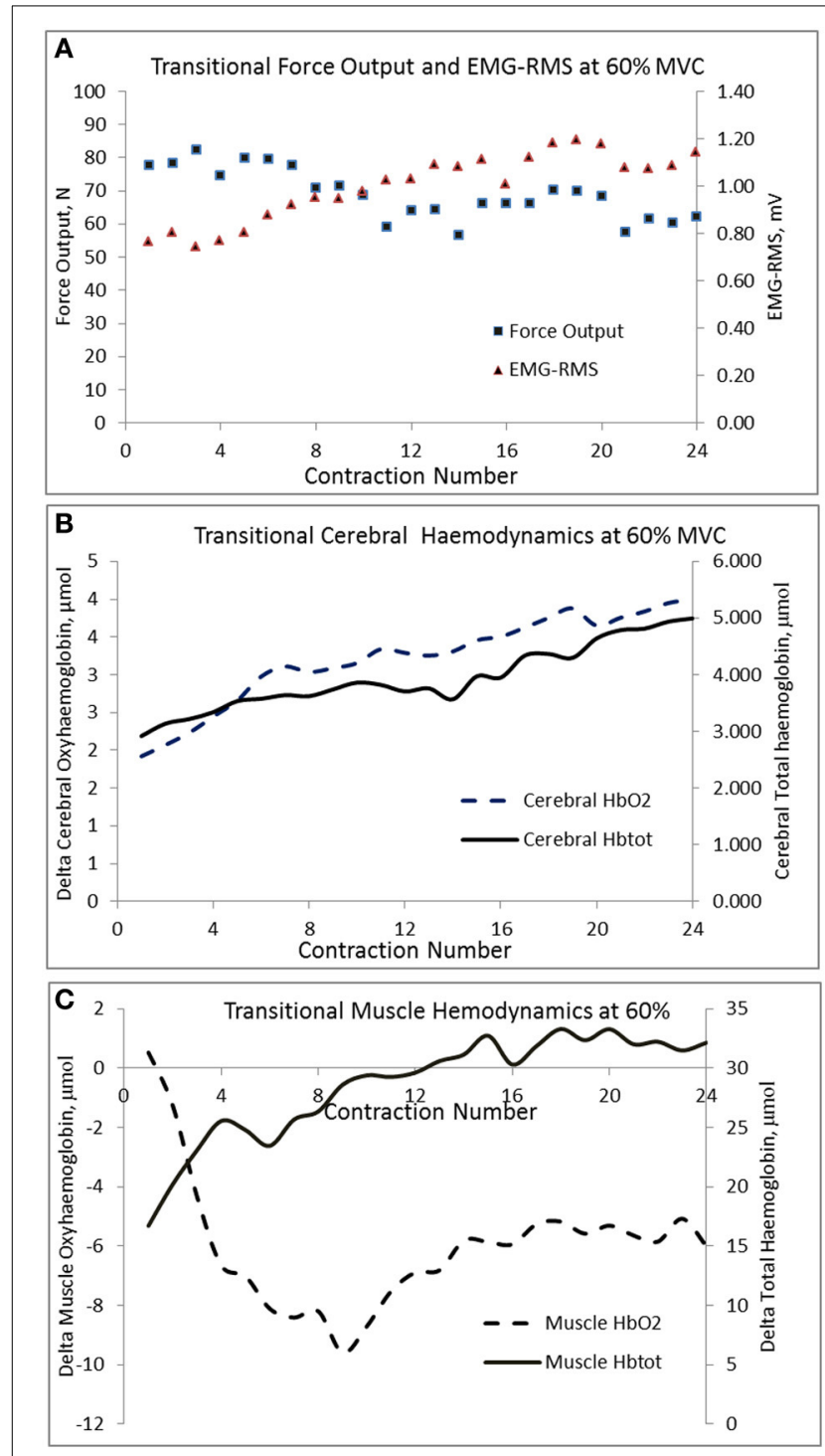

FIGURE 5 | Transitional changes in force output, cerebral, and muscle hemodynamic responses during the $\mathbf{2 4}$ contractions of the biceps brachii at $60 \% \mathrm{MVC}$ in a representative subject.

training regimens to enhance muscle strength and endurance in order to delay fatigue.

\section{CONCLUSIONS}

Intermittent isometric elbow flexion performed at 20, 40, and $60 \%$ of MVC for 2 min (24 contractions) elicited significant increases in RMS and prefrontal cortex $\mathrm{HbO}_{2}$ with concomitant decreases in $\mathrm{HHb}$. These findings are indicative of enhanced neuronal activation with increasing exercise intensity. Meanwhile, the progressive muscle $\mathrm{HbO}_{2}$ declined during increasing effort, coupled with elevations in $\mathrm{HHb}$ and Hbtot, implying greater oxygen utilization by the contracting muscle fibers. Finally, the development of muscle fatigue during intermittent isometric contraction at $60 \%$ MVC, accompanied by systematic increases in RMS, as well as cerebral $\mathrm{HbO}_{2}$ and $\mathrm{Hb}_{\text {tot }}$ with a leveling off in muscle
$\mathrm{HbO}_{2}$ and $\mathrm{Hb}_{\text {tot }}$ suggests that muscle fatigue at this intensity was partly due to reduced peripheral oxygen availability rather than impaired central neuronal activation.

\section{AUTHOR CONTRIBUTIONS}

All five co-authors actively participated in: (1) designing this project, (2) conducting the pilot studies, and (3) data collection. The experimental data were examined and evaluated by Yagesh Bhambhani and Jui-Lin Fan. The statistical analyses were completed by Yagesh Bhambhani. The first draft of the manuscript was written by Yagesh Bhambhani and reviewed by each coauthor. The comments of the co-authors were incorporated into the manuscript by Yagesh Bhambhani. The final submission to Frontiers in Physiology was completed by Yagesh Bhambhani.

\section{REFERENCES}

Ainslie, P. N., Barach, A., Murrell, C., Hamlin, M., Hellemans, J., and Ogoh, S. (2007). Alterations in cerebral autoregulation and cerebral blood flow velocity during acute hypoxia: rest and exercise. Am. J. Physiol. Heart Circ. Physiol. 292, H976-H983. doi: 10.1152/ajpheart.00639.2006

Amann, M., and Kayser, B. (2009). Nervous system function during exercise in hypoxia. High Alt. Med. Biol. 10, 149-164. doi: 10.1089/ham.2008.1105

Amann, M., Romer, L. M., Subudhi, A. W., Pegelow, D. F., and Dempsey, J. A. (2007). Severity of arterial hypoxaemia affects the relative contributions of peripheral muscle fatigue to exercise performance in healthy humans. J. Physiol. 581(pt 1), 389-403. doi: 10.1113/jphysiol.2007.129700

Bhambhani, Y. (2012). Application of near infrared spectroscopy to evaluate cerebral and muscle hemodynamics during exercise and sport. JNIRS 20, 117-139. doi: 10.1255 /jnirs. 978

Bhambhani, Y., Maikala, R., Farag, M., and Rowland, G. (2006). Reliability of near-infrared spectroscopy measures of cerebral oxygenation and blood volume during handgrip exercise in nondisabled and traumatic brain-injured subjects. J. Rehabil. Res. Dev. 43, 845-856. doi: 10.1682/JRRD.2005.09.0151

Bhambhani, Y., Malik, R., and Mookerjee, S. (2007). Cerebral oxygenation declines at exercise intensities above the respiratory compensation threshold. Respir. Physiol. Neurobiol. 156, 196-202. doi: 10.1016/j.resp.2006.08.009

Billaut, F., Davis, J. M., Smith, K. J., Marino, F. E., and Noakes, T. D. (2010). Cerebral oxygenation decreases but does not impair performance during self-paced, strenuous exercise. Acta. Physiol. (Oxf). 198, 477-486. doi: 10.1111/j.17481716.2009.02058.x

Bouillard, K., Frere, J., Hug, F., and Guevel, A. (2012). Prediction of timeto-exhaustion in the first dorsal interosseous muscle from early changes in surface electromyography parameters. Muscle Nerve 45, 835-840. doi: $10.1002 /$ mus. 23253

Boushel, R., Langberg, H., Olesen, J., Nowak, M., Simonsen, L., Bulow, J., et al. (2000). Regional blood flow during exercise in humans measured by nearinfrared spectroscopy and indocyanine green. J. Appl. Physiol. 89, 1868-1878.

Burnley, M., Vanhatalo, A., and Jones, A. M. (1985). Distinct profiles of neuromuscular fatigue during muscle contractions below and above the critical torque in humans. J. Appl. Physiol. 113, 215-223. doi: 10.1152/japplphysiol. 00022.2012

Colier, W. N., Meeuwsen, I. B., Degens, H., and Oeseburg, B. (1995). Determination of oxygen consumption in muscle during exercise using near infrared spectroscopy. Acta. Anaesthesiol. Scand. Suppl. 107, 151-155. doi: 10.1111/j.13996576.1995.tb04350.x

Dalsgaard, M. K., and Secher, N. H. (2007). The brain at work: a cerebral metabolic manifestation of central fatigue? J. Neurosci. Res. 85, 3334-3339. doi: $10.1002 /$ inr. 21274

Enoka, R. M., and Duchateau, J. (2008). Muscle fatigue: what, why and how it influences muscle function. J. Physiol. 586(pt 1), 11-23. doi: 10.1113/jphysiol.2007.139477

Fallentin, N., Jørgensen, K., and Simonsen, E. B. (1993). Motor unit recruitment during prolonged isometric contractions. Eur. J. Appl. Physiol. 67, 335-341. doi: 10.1007/BF00357632

Felici, F., Quaresima, V., Fattorini, L., Sbriccoli, P., Filligoi, G. C., and Ferrari, M. (2009). Biceps brachii myoelectric and oxygenation changes during static 
and sinusoidal isometric exercises. J. Electromyogr. Kinesiol. 19, e1-e11. doi: 10.1016/j.jelekin.2007.07.010

Gandevia, S. C. (2001). Spinal and supraspinal factors in human muscle fatigue. Physiol. Rev. 81, 1725-1789.

Gauche, E., Couturier, A., Lepers, R., Michaut, A., Rabita, G., and Hausswirth, C. (2009). Neuromuscular fatigue following high versus low-intensity eccentric exercise of biceps brachii muscle. J. Electromyogr. Kinesiol. 19, e481-e486. doi: 10.1016/j.jelekin.2009.01.006

Giller, C. A., Giller, A. M., Cooper, C. R., and Hatab, M. R. (2000). Evaluation of the cerebral hemodynamic response to rhythmic handgrip. J. Appl. Physiol. 88, 2205-2213.

Gomes, P. S., Matsuura, C., and Bhambhani, Y. N. (2013). Effects of hypoxia on cerebral and muscle haemodynamics during knee extensions in healthy subjects. Eur. J. Appl. Physiol. 113, 13-23. doi: 10.1007/s00421-012-2408-7

Gonzalez-Alonso, J., Dalsgaard, M. K., Osada, T., Volianitis, S., Dawson, E. A., Yoshiga, C. C., et al. (2004). Brain and central haemodynamics and oxygenation during maximal exercise in humans. J. Physiol. 557(pt 1), 331-342. doi: 10.1113/jphysiol.2004.060574

Haykowsky, M. J., Eves, N. D., Warburton, R. D. E., and Findlay, M. J. (2003). Resistance exercise, the Valsalva maneuver, and cerebrovascular transmural pressure. Med. Sci. Sports Exerc. 35, 65-68. doi: 10.1249/01.MSS.0000043449. 65647.C9

Henneman, E., Somjen, G., and Carpenter, O. (1965). Functional significance of cell size in spinal motoneurons. J. Neurophysiol. 28, 560-580.

Huppert, T. J., Hoge, R. D., Diamond, S. G., Franceschini, M. A., and Boas, D. A. (2006). A temporal comparison of BOLD, ASL, and NIRS hemodynamic responses to motor stimuli in adult humans. Neuroimage 29, 368-382. doi: 10.1016/j.neuroimage.2005.08.065

Kime, R., Hamaoka, T., Sako, T., Murakami, M., Homma, T., Katsumura, T., et al. (2003). Delayed reoxygenation after maximal isometric handgrip exercise in high oxidative capacity muscle. Eur. J. Appl. Physiol. 89, 34-41. doi: 10.1007/s00421-002-0757-3

Korotkov, A., Radovanovic, S., Ljubisavljevic, M., Lyskov, E., Kataeva, G., Roudas, M., et al. (2005).Comparison of brain activation after sustained non-fatiguing and fatiguing muscle contraction: a positron emission tomography study. Exp. Brain Res. 163, 65-74. doi: 10.1007/s00221-004-2141-5

Lindstrom, L., Magnusson, R., and Petersen, I. (1970). Muscle fatigue and action potential conduction velocity studied with frequency analysis of EMG signals. Electromyography 10, 341-356.

Mancini, D. M., Bolinger, L., Li, H., Kendrick, K., Chance, B., and Wilson, J. R. (1994). Validation of near-infrared spectroscopy in humans. J. Appl. Physiol. 77, 2740-2747.

Maton, B., and Gamet, D. (1989).The fatigability of two agonistic muscles in human isometric voluntary submaximal contraction: an EMG study. II: motor unit firing rate and recruitment. Eur. J. Appl. Physiol. 58, 369-374. doi: 10.1007/BF006 43511

Matsuura, C., Gomes, P. S., Haykowsky, M., and Bhambhani, Y. (2011).Cerebral and muscle oxygenation changes during static and dynamic knee extensions to voluntary fatigue in healthy men and women: a near infrared spectroscopy study. Clin. Physiol. Funct. Imaging 31, 114-123. doi: 10.1111/j.1475097X.2010.00986.x

McCully, K. K., and Hamaoka, T. (2000). Near-infrared spectroscopy: what can it tell us about oxygen saturation in skeletal muscle? Exerc. Sport Sci. Rev. 28, 123-127.

Millet, G., Martin, V., Martin, A., and Verger, S. (2011). Electrical stimulation for testing neuromuscular function: from sport to pathology. Eur. J. Appl. Physiol. 111, 2489-2500. doi: 10.1007/s00421-011-1996-y

Muthalib, M., Ferrari, M., Quaresima, V., and Nosaka, K. (2012). Frontal cortex activation during electrical muscle stimulation as revelaled by functional nearinfrared spectroscopy. Adv. Exp. Med. Biol. 15, 45-49. doi: 10.1007/978-1-46141566-4_7

Muthalib, M., Jubeau, M., Millet, G. Y., Maffiuletti, N. A., Ferrari, M., and Nosaka, K. (2010a). Biceps brachii muscle oxygenation in electrical muscle stimulation. Clin. Physiol. Funct. Imaging 30, 360-368. doi: 10.1111/j.1475097X.2010.00953.X

Muthalib, M., Lee, H., Millet, G. Y., Ferrari, M., and Nosaka, K. (2010). Comparison between maximal lengthening and shortening contractions for biceps brachii muscle oxygenation and hemodynamics. J. Appl. Physiol. 109, 710-720. doi: 10.1152/japplphysiol.01297.2009
Muthalib, M., Millet, G. Y., Quaresima, V., and Nosaka, K. (2010b). Reliability of near-infrared spectroscopy for measuring biceps brachii oxygenation during sustained and repeated isometric contractions. J. Biomed. Opt. 15:017008. doi: 10.1117/1.3309746

Neyroud, D., Maffiuletti, N. A., Kayser, B., and Place, N. (2012). Mechanisms of fatigue and task failure induced by sustained submaximal contractions. Med. Sci. Sports Exerc. 44, 1243-1251. doi: 10.1249/MSS.0b013e318245cc4d

Neyroud, D., Ruttimann, J., Mannion, A. F., Millet, G. Y., Maffiuletti, N. A., Kayser, B., et al. (2013). Comparison of neuromuscular adjustments associated with sustained isometric contractions of four different muscle groups., J. Appl. Physiol. 114, 1426-1434. doi: 10.1152/japplphysiol.01539.2012

Ogoh, S., and Ainslie, P. N. (2009).Cerebral blood flow during exercise: mechanisms of regulation. J. Appl. Physiol. 107, 1370-1380. doi: 10.1152/japplphysiol.00573.2009

Pappas, P., Asakawa, S., Delp, L., Zajac, E., and Drace, E. (2002). Non-uniform shortening in the biceps brachii during elbow flexion. J. Appl. Physiol. 92, 2381-2389. doi: 10.1152/japplphysiol.00843.2001

Payne, S. J., Mohammad, J., Tisdall, M. M., and Tachtsidis, I. (2011). Effects of arterial blood gas levels on cerebral blood flow and oxygen transport. Biomed. Opt. Express 2, 966-979. doi: 10.1364/BOE.2.000979

Pereira, M. I., Gomes, P. S., and Bhambhani, Y. N. (2007). A brief review of the use of near infrared spectroscopy with particular interest in resistance exercise. Sports Med. 37, 615-624. doi: 10.2165/00007256-200737070-00005

Place, N., Bruton, J. D., and Westerblad, H. (2009). Mechanisms of fatigue induced by isometric contractions in exercising humans and in mouse isolated single muscle fibres. Clin. Exp. Pharmacol. Physiol. 36, 334-339. doi: 10.1111/j.14401681.2008.05021.x

Pott, F., Van Lieshout, J. J., Ide, K., Madsen, P., and Secher, N. H. (2003). Middle cerebral artery blood velocity during intense static exercise is dominated by a Valsalva maneuver. J. Appl. Physiol. 94, 1335-1344. doi: 10.1152/japplphysiol.00457.2002

Praagman, M., Veeger, H. E., Chadwick, E. K., Colier, W. N., and van der Helm, F. C. (2003). Muscle oxygen consumption, determined by NIRS, in relation to external force and EMG. J. Biomech. 36, 905-912. doi: 10.1016/S00219290(03)00081-2

Prompers, J. J., Wessels, B., Kemp, G. J., and Nicolay, K. (2014). MITOCHONDRIA: investigation of in vivo muscle mitochondrial function by $\mathrm{P}$ magnetic resonance spectroscopy. Int. J. Biochem. Cell Biol. 50, 67-72. doi: 10.1016/j.biocel.2014. 02.014

Rasmussen, P., Dawson, E. A., Nybo, L., van Lieshout, J. J., Secher, N. H., and Gjedde, A. (2007). Capillary-oxygenation-level-dependent near-infrared spectrometry in frontal lobe of humans. J. Cereb. Blood Flow Metab. 27, 1082-1093. doi: $10.1038 /$ sj.jcbfm.9600416

Rasmussen, P., Nielsen, J., Overgaard, M., Krogh-Madsen, R., Gjedde, A., Secher, N. H., et al. (2010). Reduced muscle activation during exercise related to brain oxygenation and metabolism in humans. J. Physiol. 588(pt 11), 1985-1995. doi: 10.1113/jphysiol.2009.186767

Sato, K., Ogoh, S., Hirasawa, A., Oue, A., and Sadamoto, T. (2011). The distribution of blood flow in the carotid and vertebral arteries during dynamic exercise in humans. J. Physiol. 589(pt 11), 2847-2856. doi: 10.1113/jphysiol.2010. 204461

Schroeter, M. L., Kupka, T., Mildner, T., Uludag, K., and von Cramon, D. Y. (2006). Investigating the post-stimulus undershoot of the BOLD signala simultaneous fMRI and fNIRS study. Neuroimage 30, 349-358. doi: 10.1016/j.neuroimage.2005.09.048

Shibuya, K., Tanaka, J., Kuboyama, N., Murai, S., and Ogaki, T. (2004a). Cerebral cortex activity during supramaximal exhaustive exercise. J. Sports Med. Phys. Fitness 44, 215-219.

Shibuya, K., Tanaka, J., Kuboyama, N., and Ogaki, T. (2004b). Cerebral oxygenation during intermittent supramaximal exercise. Respir. Physiol. Neurobiol. 140, 165-172. doi: 10.1016/j.resp.2003.11.004

Subudhi, A. W., Miramon, B. R., Granger, M. E., and Roach, R. C. (2009). Frontal and motor cortex oxygenation during maximal exercise in normoxia and hypoxia. J. Appl. Physiol. 106, 1153-1158. doi: 10.1152/japplphysiol.91475. 2008

van Beekvelt, M. C., Borghuis, M. S., van Engelen, B. G., Wevers, R. A., and Colier, W. N. (2001). Adipose tissue thickness affects in vivo quantitative nearIR spectroscopy in human skeletal muscle. Clin. Sci. (Lond). 101, 21-28. doi: $10.1042 /$ CS20000247 
Vianna, L. C., Araujo, C. G., and Fisher, J. P. (2009). Influence of central command and muscle afferent activation on anterior cerebral artery blood velocity responses to calf exercise in humans. J. Appl. Physiol. 107, 1113-1120. doi: 10.1152/japplphysiol.00480.2009

Zebrowska, A., Gasior, Z., and Jastrzebski, D. (2013). Cardiovascular effects of the valsalva maneuver during static arm exercise in elite power lifting athletes. $A d v$. Exp. Med. Biol. 755, 335-342. doi: 10.1007/978-94-007-4546-9_42

Zwarts, M. J., and Arendt-Nielsen, L. (1988). The influence of force and circulation on average muscle fibre conduction velocity during local muscle fatigue. Eur. J. Appl. Physiol. 58, 278-283. doi: 10.1007/BF00417263

Conflict of Interest Statement: The authors declare that the research was conducted in the absence of any commercial or financial relationships that could be construed as a potential conflict of interest.
Received: 27 January 2014; accepted: 29 April 2014; published online: 11 June 2014. Citation: Bhambhani Y, Fan J-L, Place N, Rodriguez-Falces J and Kayser B (2014) Electromyographic, cerebral, and muscle hemodynamic responses during intermittent, isometric contractions of the biceps brachii at three submaximal intensities. Front. Physiol. 5:190. doi: 10.3389/fphys.2014.00190

This article was submitted to Exercise Physiology, a section of the journal Frontiers in Physiology.

Copyright (c) 2014 Bhambhani, Fan, Place, Rodriguez-Falces and Kayser. This is an open-access article distributed under the terms of the Creative Commons Attribution License (CC BY). The use, distribution or reproduction in other forums is permitted, provided the original author(s) or licensor are credited and that the original publication in this journal is cited, in accordance with accepted academic practice. No use, distribution or reproduction is permitted which does not comply with these terms. 